\title{
La regulación de la responsabilidad de cuidar y su impacto de género ${ }^{1}$
}

\section{Samara de las Heras Aguilera}

Investigadora del Grupo Antígona, Derechos y Sociedad con Perspectiva de Género y profesora del Área de Filosofía del Derecho de la Universidad Autónoma de Barcelona <samara.delasheras@uab.cat>

\begin{abstract}
Zainketen antolaketa sozialaren oinarria da, hein handi batean, bere inguruan buruturiko erregulazio juridikoa. Bertan zehazten da zein pertsonek eta zein modutan zaindu behar dituzten, eta norenak diren zainketen inguruan gertatzen diren ardura horiek. Azterlan batzuek erakusgarri jarri dute Espainian indarrean dagoen zainketen sistema oinarritzen dela lanaren banaketa sexualean, eta horren ondorioz, arlo pribatuko jarduera propiotzat ulertzen den heinean, emakumeek izaten dutela zainketen ardurarik gehien. Horren haritik, artikulu honetan zalantzan jartzen da zainketen antolakuntza juridikoaren atzean ageri den ideologia zein den. Bertan nabarmentzen dira horrek dituen ondorio sozial eta eskubideen urraketak, eta baita ere azaltzen da esparru juridiko berri bat osatzeko premia, horren bidez zainketen arduren inguruko bidezkoa izango den banaketa bat egin eta berauen egoera eta gaitasun desberdinei laguntzeko premiako dituzten arreta berezituak eskubideen bitartez bermatu ahal izatea.
\end{abstract}

\section{GAKO-HITZAK:}

Zainketak, legezko ardura, giza eskubideak, zainketaren etika, Feminismo juridikoa.
La organización social de los cuidados depende en buena medida de su regulación jurídica, que determina qué personas y de qué manera precisan ser cuidadas, y quiénes deben asumir las responsabilidades de cuidado. Diversos estudios han mostrado que el sistema de cuidados vigente en España se asienta sobre la división sexual del trabajo, que supone que la mayor parte de las responsabilidades de cuidado recaen sobre las mujeres, en cuanto actividad considerada propia del ámbito privado. En consecuencia, en el presente artículo se cuestiona la ideología que subyace en la organización jurídica de los cuidados, evidenciando las consecuencias sociales y las vulneraciones de derechos que conlleva, así como la necesidad de articular un nuevo marco jurídico que posibilite un reparto equitativo de las responsabilidades de cuidado, y garantice los derechos de las personas cuidadoras y de quienes requieren de atenciones especiales por razón de sus situaciones y capacidades diversas.

\section{Palabras Clave:}

Cuidados, responsabilidad legal, derechos humanos, ética del cuidado, Feminismo jurídico.

\footnotetext{
${ }^{1}$ Una versión previa del presente trabajo se presentó en el V Congreso de la Red Española de Política Social (REPS) ‘Desigualdad y democracia: política públicas e innovación social', celebrado el 5 y 6 de febrero de 2015 en Barcelona.
} 


\section{Introducción}

Como han evidenciado numerosos estudios feministas, las sociedades se han organizado desde hace siglos en torno a la idea de la división sexual del trabajo, que otorga a las mujeres el rol de cuidadoras y a los hombres el de trabajadores, en un sistema de organización social que responde a las imposiciones del patriarcado y del capitalismo (Hartmann, 1988). Se reproduce así el pensamiento dicotómico liberal que opone y jerarquiza dualismos como lo público y el trabajo productivo, asociado a lo masculino y considerado de mayor valor que el espacio reservado a las mujeres, el privado, ámbito de la reproducción y los cuidados (Olsen, 1990).

A pesar de las transformaciones sociales que se han venido produciendo a lo largo de las últimas décadas en España, los cuidados continúan siendo hoy en día una actividad mayoritariamente femenina y desvalorizada, ya hablemos de aquellos cotidianos o informales que se prestan en el seno de las familias o de los profesionalizados (Durán, 2006). En ese sentido es importante recordar, siguiendo a la relatora especial sobre la extrema pobreza y los derechos humanos ${ }^{2}$, que son los Estados, a través del Derecho, los que en buena medida determinan la organización social de los cuidados o, en otras palabras, a quién corresponde la responsabilidad de cuidar y quién precisa de cuidados (Sepúlveda, 2013).

Por consiguiente, en las páginas que siguen se plantean algunas reflexiones sobre la organización jurídica de los cuidados en España desde la perspectiva del Feminismo ${ }^{3}$ Jurídico, es decir, desde el enfoque de las teorías críticas que tienen como objetivo "el esclarecimiento del rol que desempeña el Derecho en el mantenimiento del patriarcado" (Facio, 1999: 17). Se pretende de ese modo analizar la regulación del sistema de cuidados en el Derecho español para evidenciar de qué manera el ordenamiento jurídico perpetúa la tradicional división sexual del trabajo y la desvalorización de los cuidados.

Cabe advertir que no se pretende desarrollar un análisis exhaustivo del marco jurídico-político en este ámbito, sino destacar ciertas asunciones y preceptos que reflejan la ideología y los valores que lo sustentan. Por otra parte, teniendo presentes las consecuencias sociales de dicho marco regulatorio, y

${ }^{2}$ La relatora es una experta independiente designada por el Consejo de Derechos Humanos de la Organización de Naciones Unidas. Se pueden consultar todos sus informes en 〈http://www.ohchr.org/ SP/Issues/Poverty/Pages/SRExtremePovertylndex.aspx〉.

3 Siguiendo la propuesta de la jurista Alda Facio (1999: 202), se utiliza el término 'Feminismo' con 'F' mayúscula (y 'movimiento feminista' y 'feminismos' como sinónimos) para hacer referencia al conjunto de feminismos, es decir, a toda teoría, pensamiento y práctica social, política y jurídica que tiene por objetivo hacer evidente y terminar con la situación de dominación y discriminación que soportan las mujeres y lograr así una sociedad más justa que reconozca y garantice la igualdad plena y el disfrute efectivo de los derechos de todos los seres humanos. especialmente su impacto de género y de derechos, se ponen de relieve algunos de los retos éticos, sociales y jurídicos más relevantes - a nuestro entender- en esta materia. En ese sentido se presta especial atención al desigual reparto de las tareas y responsabilidades de cuidado y las consecuentes vulneraciones de derechos que conlleva, en particular, respecto del derecho a la igualdad sin sufrir discriminaciones por razón de género y del derecho al bienestar integral de todas las personas, independientemente de sus circunstancias 0 condicionantes personales y sociales.

En consecuencia, se plantea la necesidad de repensar el sistema de cuidados en España. En particular, se aboga por cuestionar la atribución jurídica de las responsabilidades de cuidado a las mujeres y, por tanto, la organización jurídica y social de los cuidados, con el fin de garantizar los derechos y el bienestar integral de todas las personas, tanto de quienes requieren de cuidados por razón de su edad o de sus situaciones y capacidades diversas, como de quienes habitualmente asumen la responsabilidad de cuidar.

\section{Los cuidados: una aproximación desde las teorías feministas}

Antes de abordar la regulación de los cuidados en el Derecho vigente, cabe hacer una precisión respecto a lo que se entiende por cuidados, puesto que es un concepto por lo general indeterminado y cuya definición y alcance para la doctrina $-\mathrm{y} a$ nivel social- no son pacíficos. Dicha concreción se revela fundamental desde una perspectiva jurídica, en cuanto el diseño y aplicación de las normas y políticas públicas en esta materia dependerán en buena medida de cómo se definan los cuidados.

A modo de ejemplo de la indeterminación de este concepto podemos citar la definición contenida en el Diccionario de la lengua española ${ }^{4}$, que limita el 'cuidado' a la 'acción de cuidar', que es, a su vez, definida como "2. tr. Asistir, guardar, conservar. Cuidar a un enfermo, la casa, la ropa. U. t. c. intr. Cuidar de la hacienda, de los niños". Esa falta de concreción de lo que implican los cuidados refleja no sólo la invisibilización de unas tareas que han sido tradicionalmente relegadas al espacio privado y atribuidas a las mujeres por pretendidas razones biológicas (Facio, 1999: 44), sino también la desvalorización de lo que ha sido -y aún esconsiderado como un trabajo no productivo (Pérez Orozco y López Gil, 2011: 21-22).

En otras palabras, la dificultad a la hora de definir los cuidados y concretar qué tareas conllevan se explica por el propio sistema de dominación patriarcal, que perpetúa la división sexual del trabajo a través

${ }_{4}$ Obra de referencia de la Real Academia Española, disponible en 〈http://www.rae.es〉. 
de distintos mecanismos e instituciones - como el Derecho o el propio lenguaje- que reflejan una ideología que "explícitamente devalúa a las mujeres dándoles a ellas, a sus roles, sus labores, sus productos y su entorno social, menos prestigio y/o poder" (Facio, 1999: 21).

De ahí la importancia de abordar el estudio de los cuidados teniendo presentes los debates que se han producido en el seno del movimiento feminista en las últimas décadas (Beltrán y Maquieira, 2011), no sólo porque han evidenciado que en esa invisibilización y devaluación de las tareas y roles juzgados femeninos subyace un modelo de pensamiento que todavía supone un obstáculo a la igualdad real y efectiva y al pleno disfrute de los derechos humanos, de las mujeres como cuidadoras y también de quienes precisan esa atención o asistencia (Sepúlveda, 2013: 4). Asimismo, han aportado análisis imprescindibles para comprender la importancia de los cuidados, tanto para las personas individualmente consideradas como para el conjunto de la sociedad, y cuestionar su actual organización por el impacto de género y derechos que conlleva.

Como apunta Encarna Bodelón (2010a: 183), es posible distinguir dos grandes acercamientos a esas cuestiones: por un lado, los análisis realizados desde la sociología y la economía, que critican que el enfoque producción/reproducción no permite escapar de la dimensión mercantil patriarcal (Carrasco, 2006: 45) y, por otro, los debates en torno a la llamada 'ética del cuidado', desarrollados principalmente desde la filosofía y la psicología y que ponen el acento en el valor social de los cuidados. Como veremos, esas dos aproximaciones han sido desarrolladas por el Feminismo jurídico, disciplina desde la que se ha puesto de relieve cómo el Derecho reproduce la organización patriarcal de la sociedad en función de la división sexual del trabajo.

Sin desconocer que la multiplicidad de disciplinas, corrientes, posturas y planteamientos teóricos desde las que el Feminismo ha abordado los cuidados ha permitido visibilizar su importancia y ha enriquecido su comprensión desde un enfoque integrador, cabe advertir que, incluso en la actualidad, resulta complicado definir con exactitud qué son los cuidados y qué tareas implican.

Como señalan Amaia Pérez Orozco y Silvia López Gil (2011: 20):

En aras de la visibilidad, en ocasiones, se señala un campo excesivamente amplio que parece cubrir cualquier tipo de relación humana, convirtiendo los cuidados en un cajón de sastre en el que todo cabe. Pero, por otra parte, cuando se reduce a tareas concretas, en busca de mayor operatividad política, se deja de lado el componente afectivo/ emocional que lo caracteriza [...]. ¿Cómo podemos definir entonces el hecho de cuidar? Puede decirse que cuidar es gestionar y mantener cotidianamente la vida y la salud, hacerse cargo del bienestar físico y emocional de los cuerpos, del propio y de los otros.

La complejidad del concepto 'cuidados' se refleja en esas palabras, aún más teniendo presente que las tareas de cuidado que hacen posible el bienestar integral dependerán de las necesidades y condiciones personales y sociales de cada persona en los distintos momentos de su ciclo vital (ibídem: 21). Si bien todas las personas necesitamos ser cuidadas en algún momento de nuestras vidas, no podemos obviar que hay situaciones y circunstancias en las que los cuidados se revelan imprescindibles para garantizar una existencia digna y el disfrute de derechos tan relevantes como pueden ser el derecho a la vida o a la salud.

En ese sentido, es preciso tener presente que las necesidades de cuidado variarán en función de factores como la edad o las situaciones que originan una dependencia, más o menos severa y, asimismo, del propio sistema de cuidados establecido por el Derecho. Como explica la relatora especial sobre la extrema pobreza y los derechos humanos (Sepúlveda, 2013: 5), "la prestación de cuidados se basa en una relación entre dos partes, en muchos aspectos los derechos de los cuidadores están simbióticamente entrelazados con los derechos de las personas que reciben los cuidados: sobrecargar a los cuidadores con el trabajo doméstico no remunerado afecta la calidad de los cuidados que pueden prestar. Por tanto, cuando el trabajo doméstico no remunerado no está debidamente reconocido, valorado o apoyado por el Estado, los derechos de quienes dependen de la prestación de cuidados para su salud, vida y bienestar pueden también estar violados, en particular en hogares de bajos ingresos". Se pone el acento, de ese modo, en la centralidad de la regulación jurídica del sistema de cuidados y en la necesidad de garantizar un reparto equitativo de las responsabilidades de cuidado.

En las páginas que siguen se indaga en la regulación de los cuidados en el ordenamiento jurídico español siguiendo los postulados del Feminismo Jurídico, esto es, con la finalidad de desenmascarar el carácter patriarcal del Derecho y formular alternativas, tanto en la teoría como en la práctica jurídica, que coadyuven en el objetivo final de promover sociedades más justas e igualitarias (Facio, 1999: 60).

Con ese fin, se han desarrollado distintos métodos jurídicos feministas (Bartlett, 1990; Facio, 2004) desde los que se ha cuestionado la regulación de los trabajos de cuidado y que han orientado la presente investigación. El punto de partida, como viene siendo habitual en los análisis feministas, es el proceso de toma de conciencia sobre las necesidades y las experiencias específicas de las mujeres, en este caso, respecto a las responsabilidades de cuidado. Se cuestiona por tanto, en segundo lugar, "el papel del Derecho en la invisibilización y mantenimiento de las relaciones de poder desiguales, tanto entre 
mujeres y hombres, como respecto a otros grupos que no encajan con el modelo de lo humano asumido por la teoría jurídica tradicional”. Dicho análisis cobra especial relevancia en el asunto que nos ocupa, en cuanto permite objetar el rol de mujeres como cuidadoras y, asimismo, los limitados conceptos de autonomía y dependencia asimilados por los ordenamientos jurídicos. Por último, a través del razonamiento jurídico feminista, se ponen de relieve "las injusticias y discriminaciones que los propios ordenamientos jurídicos amparan”, rebatiendo de ese modo la supuesta neutralidad del Derecho (Laporta y De las Heras, 2014).

\section{El sistema de cuidados en el ordenamiento jurídico español}

El análisis del ordenamiento jurídico español permite constatar que la regulación jurídica y política de los cuidados consolida todavía hoy la división sexual del trabajo.

En primer lugar, cabe hacer referencia al mantenimiento de la separación de las esferas pública y privada, así como la invisibilización de esta última5. A modo de ejemplo podemos citar la Constitución española, y en particular el artículo 9.2, que establece que "corresponde a los poderes públicos promover las condiciones para que la libertad y la igualdad del individuo y de los grupos en que se integra sean reales y efectivas; remover los obstáculos que impidan o dificulten su plenitud y facilitar la participación de todos los ciudadanos en la vida política, económica, cultural y social”. Se omite así la consideración del ámbito privado como un espacio de participación de la ciudadanía y de responsabilidad de las distintas instituciones del Estado.

Tampoco se nombra el concepto ‘cuidados' en ninguno de los preceptos de la norma suprema, aunque sí se alude a la "asistencia de todo orden" que los padres deben prestar a sus hijos e hijas durante su minoría de edad y en los demás casos en que legalmente proceda (artículo 39 de la Constitución, enmarcado en el capítulo III, dedicado a los principios rectores de la política social y económica). Es digno de mención que se vincule esa asistencia integral, que podríamos entender como sinónimo de cuidados, al ámbito familiar y a una situación concreta: la niñez.

La vinculación de los cuidados al ámbito de la familia ${ }^{6}$ no sólo la encontramos en dicho precepto,

${ }^{5}$ La división y jerarquización de las esferas pública y privada ha ocupado un lugar central en los estudios feministas, ya que, en palabras de Carol Pateman (1996: 33), dicha dicotomía “oculta la sujeción de las mujeres a los hombres dentro de un orden aparentemente universal, igualitario e individualista”. Desde el Feminismo Jurídico se ha cuestionado esa lógica patriarcal y el papel del Derecho en su perpetuación (Olsen, 1990; MacKinnon, 1995; Facio, 1999; Bodelón, 2010a; Igareda, 2012).

${ }^{6}$ Asimismo, es preciso apuntar que aunque la Constitución no establece explícitamente responsabilidades familiares respecto a las también en el artículo 50 se hace referencia a las obligaciones familiares respecto a otro colectivo específico, las personas mayores, en los siguientes términos:

Los poderes públicos garantizarán, mediante pensiones adecuadas y periódicamente actualizadas, la suficiencia económica a los ciudadanos durante la tercera edad. Asimismo, y con independencia de las obligaciones familiares, promoverán su bienestar mediante un sistema de servicios sociales que atenderán sus problemas específicos de salud, vivienda, cultura y ocio.

Como decíamos, el ordenamiento jurídico español perpetúa la división sexual del trabajo en primer lugar al invisibilizar el ámbito privado y las actividades que tradicionalmente han sido relegadas a este espacio $\mathrm{y}$, en segundo lugar, al considerar los cuidados como una responsabilidad de las familias, como una actividad no productiva (Igareda, 2012: 185).

Ambas cuestiones están íntimamente relacionadas. No debemos olvidar que una de las funciones básicas del Derecho es "instaurar o contribuir a asentar -junto a otras instituciones y mecanismos estabilizadores- un determinado orden en una determinada sociedad" (Díaz García, 1998: 128). En cuanto sistema de organización social, regula aquellas actividades que se consideran importantes y cuando "mantiene una postura o posición de no intervención”, transmite una idea de insignificancia, de ausencia de valor para la sociedad (Olsen, 1990: 463).

La invisibilización de los cuidados en la Constitución española responde a su consideración como una actividad propia de las mujeres, que integra el trabajo doméstico no remunerado. Se reproduce de esa manera el denominado modelo tradicional del sistema de cuidados, que "se basa fundamentalmente en la familia y el apoyo informal que, por otra parte, no dejan de ser con frecuencia eufemismos de mujer" (Mier et al., 2007: 29).

Frente a dicha desconsideración de los trabajos de cuidados, que refleja la asunción de la ideología patriarcal por el Estado y el Derecho (Facio, 1999: 48-49), desde distintas posturas feministas se ha evidenciado su importancia. Por un lado, se ha argumentado que los trabajos de cuidado son imprescindibles para posibilitar la supervivencia y el bienestar integral de todas las personas y del conjunto de la sociedad (Carrasco, 2006: 61). Es

personas en situación de dependencia, tradicionalmente la atención a este colectivo se han basado en "una cultura del cuidado fuertemente familista, una protección social débil y reforzadora de la parentela como proveedora, y una escasa asunción de responsabilidades domésticas por parte de los hombres” (Martínez Buján, 2011: 103). A pesar de las medidas sociales y las prestaciones económicas introducidas por la Ley 39/2006, de 14 de diciembre, de Promoción de la Autonomía Personal y Atención a las personas en situación de dependencia, diversos estudios señalan que buena parte de las responsabilidades de cuidado de las personas en situación de dependencia continúan siendo asumidas por las familias (Martínez Buján, 2011; Guzmán, Moscoso y Toboso, 2010). 
decir, los trabajos de cuidado inciden directamente en los derechos humanos de aquellas personas que los necesitan por su situación o sus condicionantes personales y sociales. Y, como ya se ha señalado, repercuten también en los derechos de las mujeres, en tanto que son quienes han asumido mayoritariamente la responsabilidad de cuidar (Sepúlveda, 2013).

Por otro, en términos económicos, se ha denunciado la falsedad de la separación de lo público/productivo y lo privado/reproductivo, evidenciando que el primero "descansa y se apoya en otros sectores o actividades que caen fuera de la órbita mercantil" (Carrasco, 2006: 40), así como la centralidad de los cuidados en el desarrollo social y en el crecimiento económico (Sepúlveda, 2013: 4). De ahí la reivindicación del término "trabajo de cuidados" (Carrasco, 2006: 41-46) y de la necesidad de incorporar, en palabras de María Ángeles Durán (2001: 8), este "trabajo sin precio, pero no sin valor" a los sistemas de contabilidad nacional, con el "propósito de, por un lado, representar más rigurosamente la actividad económica generadora de riqueza y, por otro lado, reivindicar la aportación, hasta ahora infravalorada, de las mujeres" (Tobío et al., 2010: 29).

Dichas reivindicaciones no se limitan al Estado español, responden a una pauta global, como podemos deducir de las palabras de la relatora especial sobre la extrema pobreza y los derechos humanos (Sepúlveda, 2013: 3) cuando denuncia que:

\section{El trabajo doméstico y el cuidado de otras personas se han mantenido en gran parte invisibles en las políticas, las estadísticas, los cálculos económicos, y el discurso político, y están generalmente subvalorados por la sociedad y los formuladores de política, pese a que su valor monetario se calcula que representa entre $10 \mathrm{y}$ más de $50 \%$ del PIB.}

En definitiva, diversas voces feministas han cuestionado la división y la jerarquización del trabajo productivo y reproductivo consolidadas por los ordenamientos jurídicos y han defendido la revalorización de cuidados, objetando al mismo tiempo su organización social (Pérez Orozco, 2011: 40-42). De ese modo, se ha visibilizado su importancia tanto a nivel individual, como en términos de desarrollo social y económico, desbordando así "el mundo de la familia y de lo interpersonal, reconociéndose los contextos, las iniciativas y las políticas que configuran formas variadas de atender a los que necesitan ser cuidados" (Tobío et al., 2010: 26-27).

Esas críticas y consideraciones han tenido un cierto impacto en los ordenamientos jurídicos, especialmente en los últimos años, en los que el debate sobre la organización de los cuidados está cobrando relevancia por el actual contexto social y económico (Mier et al., 2007: 30). En el plano internacional, podemos citar el reconocimiento del valor social de los cuidados en la Convención para la eliminación de todas las formas de discriminación contra la mujer (en adelante, CEDAW), adoptada por la Organización de Naciones Unidas en 1979 y ratificada por el Estado español en 1984. La CEDAW, norma vinculante considerada la carta de los derechos de las mujeres, reconoce en su preámbulo no sólo "el gran aporte de la mujer al bienestar de la familia y al desarrollo de la sociedad, hasta ahora no plenamente reconocido", sino también el impacto de género que conlleva, al afirmar que "para lograr la plena igualdad entre el hombre y la mujer es necesario modificar el papel tradicional tanto del hombre como de la mujer en la sociedad y en la familia".

Con ese fin, insta a los Estados a adoptar "todas las medidas apropiadas para: a) Modificar los patrones socioculturales de conducta de hombres y mujeres, con miras a alcanzar la eliminación de los prejuicios y las prácticas consuetudinarias y de cualquier otra índole que estén basados en la idea de la inferioridad o superioridad de cualquiera de los sexos o en funciones estereotipadas de hombres y mujeres" (artículo 5), así como a "alentar el suministro de los servicios sociales de apoyo necesarios para permitir que los padres combinen las obligaciones para con la familia con las responsabilidades del trabajo y la participación en la vida pública, especialmente mediante el fomento de la creación y desarrollo de una red de servicios destinados al cuidado de los niños" (artículo 11.2.c).

A pesar de los reproches que merece la vinculación de los cuidados únicamente con la infancia, cuestión a la que se hará referencia a continuación, lo cierto es que en esa norma podemos encontrar un reconocimiento no sólo de la función social de los cuidados, sino también de la necesidad de redistribuir las responsabilidades de cuidado tanto en el seno de las familias, como en el ámbito de acción del Estado a través de los servicios sociales.

A nivel estatal, podemos mencionar la Ley 39/2006, de 14 de diciembre, de Promoción de la Autonomía Personal y Atención a las Personas en Situación de Dependencia (en adelante, LAPAD), que explícitamente reconoce en su preámbulo que "hasta ahora, han sido las familias, y en especial las mujeres, las que tradicionalmente han asumido el cuidado de las personas dependientes, constituyendo lo que ha dado en llamarse el 'apoyo informal'" y añade que el contexto social actual convierte la atención a este colectivo en "un reto ineludible para los poderes públicos, que requiere una respuesta firme, sostenida y adaptada al actual modelo de nuestra sociedad”.

Por otra parte, la Ley Orgánica 3/2007, de 22 de marzo, para la Igualdad Efectiva de Mujeres y Hombres también reconoce "el valor del trabajo de las mujeres, incluido el doméstico" y los derechos de conciliación de la vida personal, familiar y laboral 
"de forma que fomenten la asunción equilibrada de las responsabilidades familiares evitando toda discriminación basada en su ejercicio" (artículo 44).

Si bien en esas normas se intuye un cambio de paradigma respecto a la organización jurídica de los cuidados, cabe cuestionar la ideología liberal que subyace en esos preceptos (Pateman, 1996), por la asunción de que sólo se requiere de cuidados en situaciones concretas, como son la niñez, la vejez o las situaciones de dependencia. En otras palabras, el ordenamiento jurídico parte de la presunción de que las personas somos autónomas y la necesidad de ser cuidado/a es un hecho excepcional. Es más, “normalmente, el término 'cuidado' va unido al de 'dependencia”' (Carrasco, 2006: 53), tal como podemos observar en el artículo 2 de la LAPAD, que concreta esos conceptos.

Así, define la autonomía como: "la capacidad de controlar, afrontar y tomar, por propia iniciativa, decisiones personales acerca de cómo vivir de acuerdo con las normas y preferencias propias así como de desarrollar las actividades básicas de la vida diaria" y limita la dependencia al "estado de carácter permanente en que se encuentran las personas que, por razones derivadas de la edad, la enfermedad o la discapacidad, y ligadas a la falta o a la pérdida de autonomía física, mental, intelectual o sensorial, precisan de la atención de otra u otras personas 0 ayudas importantes para realizar actividades básicas de la vida diaria o, en el caso de las personas con discapacidad intelectual o enfermedad mental, de otros apoyos para su autonomía personal”.

Cabe llamar la atención sobre el hecho de que la dependencia se vincule a situaciones concretas y con un carácter permanente, lo que excluye todas aquellas realidades en las que una persona puede requerir cuidados de una manera puntual. Por otra parte, se ha criticado que "adopta un concepto de situación de dependencia centrado únicamente en las limitaciones de la persona, que olvida totalmente las restricciones sociales que intervienen igualmente en la situación de dependencia, y en consecuencia ignora la interacción entre ambos factores" (Guzmán et al., 2010: 47).

Del mismo modo, cabe cuestionar la definición de autonomía contenida en dicha norma, puesto que como explica Eduardo Díaz Velázquez (2010: 34-35), se restringe a la capacidad de decidir y realizar las actividades básicas de la vida diaria, obviando así otras actividades instrumentales y avanzadas fundamentales, "como serían el acceso a la educación y al empleo, la administración del dinero y el desarrollo de responsabilidades (importante, sobre todo, para establecer apoyos a las personas con discapacidades intelectuales), la participación social y política (participación pública), el desarrollo de las habilidades sociales, la movilidad en el entorno o el disfrute del tiempo libre, entre otras". En ese sentido, se ha denunciado que la LAPAD no promueve una autonomía personal plena (ibídem:
36), entre otras razones, porque se basa en un enfoque que se asienta sobre un modelo médico, en lugar de optar por un modelo social (Guzmán et al., 2010: 53), que "antepone el asistencialismo a la inclusión real” (Díaz Velázquez, 2010: 37).

\section{En palabras de Amaia Pérez Orozco y Silvia López} Gil, “el debate se juega en términos filosóficos entre comprender el cuidado como parte de la vida humana o, por el contrario, como excepción en la misma; dicho de otro modo, entre asumir la vulnerabilidad y la dependencia de los cuerpos como elementos constitutivos de la existencia, o mantenerlos como entes aislados entre sí, dentro de un marco estricto de autonomía e independencia corporal y subjetiva" (2011: 21). Se pone el acento, de ese modo, en la idea de que todas las personas precisamos -o podemos precisar- ser cuidadas en distintos momentos de nuestra vida, por diversas situaciones y condicionantes, que harán imprescindibles unos cuidados que den respuesta a las necesidades específicas en cada contexto. Subyace en dicha propuesta el cuestionamiento del modelo de lo humano que asume el marco jurídico-político, que responde, de nuevo, a la ideología liberal y patriarcal que nos encasilla como personas autónomas, dependientes o cuidadoras?

Esa idea se puede ver reflejada en el hecho de que la garantía de la igualdad real y de los derechos de las mujeres y de otros colectivos que no encajan en ese paradigma de lo humano ${ }^{8}$ (Facio, 1999: 23) ha precisado la adopción de normas específicas como pueden ser la CEDAW $^{9}$ o la Ley Orgánica 3/2007, de 22 de marzo, para la Igualdad Efectiva de Mujeres y Hombres, en el plano estatal. Se comienza a admitir, por tanto, que "los sujetos, en cuanto categorías sociales y políticas, son contingentes, construidos, parciales, heterogéneos y contestables" (Igareda y Cruells, 2014: 5).

Sin embargo, la aprobación de normas específicas, tanto respecto de la igualdad como en materia de cuidados, apenas ha incidido en su organización social, que sigue respondiendo a la división sexual del trabajo. Así se constata, por un lado, en los datos estadísticos que año tras año muestran la

\footnotetext{
7 En palabras de Simone de Beauvoir, "la mujer se determina y se diferencia con respecto al hombre y no a la inversa; ella es lo inesencial frente a lo esencial. Él es el Sujeto, es el Absoluto: ella es la Alteridad" (2002: 50). Como se señalaba, el Derecho reproduce ese esquema al considerar que "el hombre es la referencia implícita para lo humano, la masculinidad la medida del derecho a la igualdad" (MacKinnon, 1995: 300).

${ }^{8}$ Podemos citar, a modo de ejemplo, a las personas en situación de dependencia, a las migrantes o a las menores de edad, colectivos que también han precisado de normas y políticas públicas específicas para una efectiva garantía de sus derechos, teniendo en cuenta sus diversas necesidades y situaciones.

9 En ese sentido, cabe recordar que el reconocimiento de la titularidad de los derechos humanos a las mujeres se hace a través del conocido como proceso de especificación, "fenómeno que produce serias mutaciones en el modelo occidental inicial" y que supone "una diferencia con los modelos genéricos de destinatarios de derechos fundamentales" (Peces-Barba, 1999: 180-182).
} 
feminización de los cuidados ${ }^{10}$; por otro, en los diversos estudios que evidencian que las normas y políticas públicas adoptadas no han logrado un reparto equitativo de la responsabilidad de cuidar ni, tampoco, erradicar los roles de género que aún conllevan una discriminación para las mujeres en cuanto cuidadoras (Igareda, 2012; Durán, 2006; Tobío et al., 2010).

En definitiva, podemos concluir que el ordenamiento jurídico español coadyuva en el mantenimiento de la división sexual del trabajo, entre otras, por las razones expuestas: en primer lugar, consolida la separación y jerarquización de lo público y lo productivo, frente a lo privado y lo reproductivo. Se perpetúa de ese modo una ideología que presupone que la necesidad de ser cuidado/a es excepcional y que los trabajos de cuidados son una responsabilidad familiar y, en concreto, de las mujeres. Si bien es cierto que se puede constatar un cambio de paradigma en los ordenamientos jurídicos en los últimos años y, en particular, la ampliación de los sujetos de Derecho y la consideración de sus necesidades concretas, el Derecho todavía invisibiliza su valor social y su centralidad para el sostenimiento de una vida digna y plena.

\section{Repensando la organización jurídica y social de los cuidados}

Los análisis y críticas desarrollados por las juristas feministas han visibilizado, por un lado, la influencia de lo que Carol Pateman (1996: 50) denomina el "patriarcalismo liberal" en el Derecho y, por otro, sus consecuencias en la vida de las mujeres. En ese sentido, plantean retos jurídico-políticos, sociales y éticos al desvelar la incoherencia de un Estado que se proclama Social pero delega la responsabilidad de cuidar esencialmente en las mujeres (Igareda, 2012: 186), consolidando así las discriminaciones que conlleva la división sexual del trabajo, especialmente en un contexto de crisis como el actual.

Por ello, se subraya la necesidad de "situar la problemática del cuidado en el centro de la discusión acerca del Estado del bienestar y los procesos de reestructuración que está experimentando en la actualidad” (Tobío et al., 2010: 27). Esa pretensión supone varios desafíos filosóficos, entre los que cabe mencionar la reconceptualización radical de los cuidados, que cuestione tanto esa noción $-\mathrm{y}$, con ello, los conceptos de autonomía y dependenciacomo su organización social, poniendo el acento en el reparto equitativo de la responsabilidad de cuidar.

En cuanto a la primera cuestión, cabe recordar que "la filosofía moral moderna, y en concreto las teorías universalistas de la justicia, han acentuado nuestro valor como personas morales a costa del

\footnotetext{
${ }^{10}$ Todos los datos relativos a los cuidados pueden consultarse en la página web del Instituto de la Mujer [<http://www.inmujer.gob.es/ estadisticas/mujeresHombres/home.htm〉].
}

olvido y la represión de nuestra vulnerabilidad y dependencia como seres corporales" (Benhabib, 1992: 49). Frente a la ficción del sujeto autónomo liberal, desde diversas posturas feministas se ha reivindicado la interdependencia de todas las personas (Pérez Orozco, 2011: 44). Como recuerda María Ángeles Durán, incluso “dentro de las familias se producen divisiones del trabajo, generalmente siguiendo un eje de género, que hace a las mujeres 'dependientes' respecto a los ingresos que son aportados por los varones, y a los varones les hace igualmente 'dependientes' respecto a los servicios no remunerados que producen las mujeres para el hogar” (Durán, 2006: 62).

En definitiva se defiende que, de distinto modo y por diversas circunstancias, todas las personas necesitaremos ser cuidadas en algún momento de nuestras vidas, cuestionando así las concepciones tradicionales de autonomía y dependencia y, en última instancia, el propio sistema de cuidados.

Respecto a ese segundo reto, la reorganización jurídica y social de los cuidados, es preciso recordar que a pesar del cambio de modelo social que ha supuesto la participación de las mujeres en los espacios públicos (y, en concreto, de la incorporación masiva al ámbito laboral), así como su consideración como ciudadanas y titulares de derechos, las mujeres continúan siendo de hecho las responsables de los cuidados.

Como apunta Lucía Martínez Virto (2010: 223), éste es uno de los mayores desafíos de los Estados europeos, especialmente de aquellos en los que "el peso de los cuidados personales sigue recayendo en las familias, las cuales son la base sobre la que los Estados de Bienestar de estos países se sostienen", como sucede en España. En ese sentido, diversos estudios muestran que a pesar de la adopción de normas en materia de conciliación de la vida personal, familiar y laboral y de la creación del Sistema para la Autonomía y Atención a la Dependencia, las familias y, en particular las mujeres, continúan siendo el soporte esencial en la provisión de cuidados (Mier et al., 2007; Martínez Virto, 2010; Tobío et al., 2010; Martínez Buján, 2011).

Como explica Raquel Martínez Buján (2011: 101 y 103), en la actualidad la mayor parte de los cuidados en el ámbito informal o familiar continúan siendo asumidos por las mujeres de manera no remunerada ni regulada. Se mantiene así "una cultura del cuidado fuertemente familista, una protección social débil y reforzadora de la parentela como proveedora, y una escasa asunción de responsabilidades domésticas por parte de los hombres".

Ese sistema de cuidados, basado en su consideración como una actividad no productiva y no remunerada que debe ser gestionada en el ámbito familiar, tiene un claro impacto en la vida y los derechos de las mujeres: por un lado, por la carga de trabajo desproporcionada que conlleva para las cuidadoras 
(Pérez Orozco, 2011: 36-37) y, por otro, porque ahonda la feminización de la pobreza y de la precariedad (Sepúlveda, 2013: 6). Cabe apuntar que esas consecuencias afectan tanto a las mujeres que asumen las responsabilidades de cuidado de sus familiares de manera no remunerada ${ }^{11}$, como a quienes trabajan en el sector del servicio doméstico, también sumamente feminizado y precarizado (Martínez Buján, 2011) ${ }^{12}$.

En resumen, desde distintas disciplinas y ámbitos, se ha mostrado cómo el hecho de que se atribuya a las mujeres la responsabilidad de cuidar (y el trabajo doméstico, en general) supone un obstáculo a la igualdad por razón de sexo y al pleno disfrute de los derechos humanos de las cuidadoras y de quienes precisan de cuidados.

Es necesario, por tanto, repensar la organización social de los cuidados, teniendo presente de una manera transversal una perspectiva feminista y de derechos. Este último enfoque es fundamental a la hora de considerar su reorganización no sólo porque permite "analizar las desigualdades que se encuentran en el centro de los problemas de desarrollo y corregir las prácticas discriminatorias y el injusto reparto del poder que obstaculizan el progreso" (Oficina del Alto Comisionado de Naciones Unidas para los Derechos Humanos, 2006: 15), sino argumentar que la responsabilidad de garantizar los derechos y el bienestar integral de todas las personas corresponde a los Estados y al conjunto de la sociedad. En ese sentido, se sostiene que "para que sea posible el disfrute de los derechos, tanto de quienes prestan cuidados, como de quienes los reciben, el costo de dichos cuidados debe ser asumido por la sociedad de manera más amplia” (Sepúlveda, 2013: 5).

De ese modo, se reclama la necesidad de desarrollar un nuevo marco de relaciones de ciudadanía, que desde una perspectiva feminista implicaría, siguiendo a Encarna Bodelón (2010b: 21), superar el pensamiento patriarcal que propugna la división de lo público y lo privado, tener presentes la pluralidad y diversidad de capacidades, situaciones y necesidades a la hora de construir ese nuevo modelo y, por último, "unas relaciones de ciudadanía que se articulen desde una estructura de derechos que permita reconocer la vinculación de los seres humanos y sus necesidades de relación y cuidado". Se reivindica así la asunción de una ética del cuidado, que "nos guía para actuar con cuidado en

${ }^{11}$ Diversos estudios han puesto de relieve que la asunción de las responsabilidades de cuidado en el ámbito familiar de manera no remunerada incide directamente en la dependencia económica y la desprotección social de las cuidadoras, limitando su autonomía y su salud (Durán, 2006; Carrasco, 2006; Sepúlveda, 2013).

${ }^{12}$ Como explica Raquel Martínez Buján, las limitaciones y carencias de la red pública de servicios sociales y el propio contexto social y económico han propiciado una tendencia al alza en la privatización o mercantilización del servicio doméstico, a menudo prestado por mujeres inmigrantes que trabajan en condiciones precarias, entre otras razones por el "mantenimiento de un régimen laboral obsoleto, discriminante y precario basado en el paternalismo y la concepción androcéntrica de la profesión" (Martínez Virto, 2010: 227). el mundo humano y recalca el precio que supone la falta de cuidado" (Gilligan, 2014: 34). En ese sentido, Carol Gilligan (ibídem: 31 ) defiende que:

El feminismo guiado por una ética del cuidado podría considerarse el movimiento de liberación más radical -en el sentido de que llega a la raízde la historia de la humanidad. Al desprenderse del modelo binario y jerárquico del género, el feminismo no es un asunto de mujeres, ni una batalla entre mujeres y hombres, sino el movimiento que liberará a la democracia del patriarcado.

Dichas pretensiones cobran especial relevancia en el actual contexto de crisis, que viene a agravar las consecuencias sociales del sistema de organización de los cuidados vigente en España. Respecto a esa última cuestión, Amaia Pérez Orozco argumenta que aunque "la mirada hegemónica que se nos impone para pensar la crisis posiciona en el centro de atención a los mercados financieros [...], estamos afrontando una crisis sistémica y civilizatoria, en la que lo que necesitamos cuestionar es el conjunto del 'proyecto modernizador', la idea misma de desarrollo, progreso y crecimiento” (2011: 30-31). Es, en definitiva, una crisis multidimensional que plantea retos éticos y sociales primordiales y precisa de una respuesta integral y coherente de los Estados a través de las políticas públicas y el Derecho.

Sin embargo, a pesar de las profundas transformaciones sociales y jurídicas que se han producido a lo largo de las últimas décadas, el Estado de Bienestar español continúa reproduciendo la división sexual del trabajo (Igareda, 2012: 195), con las consecuencias que ese sistema conlleva. Al margen de las vulneraciones de derechos a las que ya se ha hecho mención (entre ellas el derecho a no ser discriminada por razón de género) cabe destacar otra por el desafío político que representa: su impacto demográfico y social.

El desigual reparto de la responsabilidad de cuidar y la dejación de responsabilidades por parte del Estado para erradicar los patrones sociales que obstaculizan el pleno disfrute de los derechos y fortalecer el sistema de protección social, devienen asuntos ineludibles en el actual contexto. La difícil tarea de conciliar la vida personal y familiar con las actividades desarrolladas en el ámbito laboral y otros espacios públicos, ha tenido como resultado un retraso en la edad de la maternidad y la paternidad ${ }^{13} \mathrm{y}$ una importante disminución de la tasa de fecundidad (Delgado et al., 2006: 199), con el consiguiente envejecimiento de la población y un imparable aumento de las personas en situación de dependencia (Durán, 2006: 57).

${ }^{13}$ En ese sentido, cabe recordar que año tras año ha ido aumentando la edad media del primer embarazo. Según los datos estadísticos del INE, la edad media de maternidad era 28,86 en 1990 , cifra que ha aumentado paulatinamente hasta alcanzar los 31,56 años en 2012 [〈http://www.inmujer.gob.es/estadisticas/consulta.do?area=1〉]. 
La distorsión entre la edad 'adecuada' para la maternidad y la paternidad desde una perspectiva biológica y desde una perspectiva social, responde a los "problemas derivados de la frecuente superposición de las viejas responsabilidades de cuidado atribuidas a las mujeres y las nuevas exigencias de su actividad laboral" (Tobío et al., 2010: 91) y tiene consecuencias tanto individuales como sociales. Respecto a las primeras, se han denunciado las implicaciones que tienen en la vida de las mujeres las dobles jornadas de trabajo, las cadenas de cuidado (Pérez Orozco y López Gil, 2011) o, como se ha mencionado, las dificultades para decidir libre y responsablemente cuando y cuántos hijos/as tener. Aunque esa decisión - así como "disponer de la información, la educación y los medios necesarios para poder hacerlo"- es un derecho reconocido en el Programa de Acción de la Conferencia Internacional sobre la Población y el Desarrollo celebrada en El Cairo en 1994, es posible constatar la persistencia de factores sociales, económicos y jurídicos que la obstaculizan. Entre ellos cabe destacar la persistencia de los roles de género que atribuyen a las mujeres el papel de cuidadoras y las consiguientes dificultades que encuentran para conciliar su vida personal, familiar y laboral (Esping-Andersen, 2013).

Junto a las diversas discriminaciones y vulneraciones de derechos que ese contexto provoca, la organización social de los cuidados tiene, como decíamos, un impacto social. Se ha apuntado que "España registra una tasa de fecundidad de 1,3 hijos por mujer, lo que hace que se encuentre entre los países de más baja fecundidad del mundo desarrollado" y que no se asegure el reemplazo de las generaciones (Delgado et al., 2006: 217). En consecuencia, se produce el envejecimiento y disminución de la población y el aumento de las necesidades de cuidado (Esping-Andersen, 2013: 26), pautas que ya es posible constatar en los Estados occidentales ${ }^{14} \mathrm{y}$ en España, en particular (Durán, 2006; Tobío et al., 2010).

En definitiva, esas razones - que no son todas las que cabría argumentar- justifican repensar la actual organización social de los cuidados y adoptar una nueva regulación jurídica que garantice una adecuada atención a todas las personas, dando respuesta a las necesidades específicas que requiere su situación y contexto y un reparto equitativo de la responsabilidad de cuidar.

${ }^{14}$ En ese sentido, la experta independiente de la Organización de las Naciones Unidas sobre el disfrute de todos los derechos humanos por parte de las personas de edad, ha recordado que es necesario tener presente la "revolución demográfica" que está en marcha, en particular, el envejecimiento de la población, para atender adecuadamente las necesidades de la población de mayor edad. Para profundizar en la propuesta de dicha experta, se puede consultar la nota de prensa publicada el 1 de octubre de 2014 [/http://www.ohchr.org/SP/ NewsEvents/Pages/DisplayNews.aspx?NewsID=15127\&LangID=S〉].

\section{Hacia otro sistema de cuidados: algunas conclusiones y propuestas}

Si bien se ha explicado cómo el Derecho asume una ideología patriarcal en determinadas cuestiones, en concreto a la hora de regular los cuidados, puede ser asimismo considerado una herramienta para transformar prácticas sociales a través de la adopción de normas y políticas públicas (Facio, 1999). Partiendo de esa premisa, cabe cuestionarse cómo deberían organizarse jurídica y socialmente los cuidados y, en particular, quiénes y en qué medida deberían tener la responsabilidad de cuidar.

Debemos tener presente que "España se constituye en un Estado social y democrático de Derecho, que propugna como valores superiores de su ordenamiento jurídico la libertad, la justicia, la igualdad y el pluralismo político" (artículo 1 de la Constitución), lo que implica que "el derecho a la igualdad de mujeres y hombres es un principio y un derecho fundamental exigible, y donde al Estado se le exige una actuación tendente a garantizar un nivel mínimo de bienestar en el que las necesidades básicas de la ciudadanía están satisfechas" (Igareda, 2012: 202-203). Esa obligación del Estado se concreta, como explica la relatora especial sobre la extrema pobreza y los derechos humanos en la adopción de "un enfoque general y holístico, que tenga en cuenta las necesidades y el bienestar, tanto de las personas encargadas de prestar cuidados, como de las que los reciben, al formular políticas y abordar el disfrute de derechos por ambos grupos. Antes de aplicar nuevas políticas públicas se deberá evaluar su efecto en la calidad, cantidad, intensidad y distribución del trabajo doméstico no remunerado" (Sepúlveda, 2013: 23-24).

Destaca, en primer lugar, la importancia de tomar en consideración las necesidades básicas de cada persona y superar el modelo de lo humano que universaliza el Derecho y que no responde a la diversidad de situaciones, contextos y necesidades. En palabras de Noelia Igareda y Marta Cruells, habría que "intentar descentrar cuando sea posible a los sujetos identitarios excesivamente fijados (mujeres, personas de color, grupos étnicos, etc.) para legislar en función de categorías como el género, la raza, la edad, la clase o el estatus social" (Igareda y Cruells, 2014: 9).

Dicho ejercicio de reconceptualización debería extenderse a otras nociones, como se ha defendido en el presente trabajo. Así, es fundamental repensar el alcance de las 'necesidades básicas', puesto que como recuerda Noelia Igareda actualmente los cuidados no se identifican como necesidades básicas en nuestro ordenamiento jurídico (2012: 202). 0 , como se ha sostenido, de las nociones de autonomía, dependencia y de los trabajos de cuidado, enfrentando así la ideología que subyace en las normas y, al mismo tiempo, evidenciando el valor individual y social de los cuidados. 
En ese sentido, cabe proponer una ética o una concepción de la Justicia que, como expresa Carol Gilligan, cuestione la propia raíz de los modelos de organización social: “La voz 'diferente' -aquella que oí por primera vez al escuchar a mujeres- unía la razón con la emoción, y al Yo con las relaciones. En su narrativa, las vidas de la gente estaban conectadas y eran interdependientes. Desde este punto de vista, lo contrario de la dependencia era el aislamiento" (Gilligan, 2014: 42). En definitiva, se proponen nuevas definiciones que reflejen situaciones y necesidades invisibilizadas por el Derecho, "nuevos conceptos jurídicos para traducir la situación de opresión que sufren las mujeres en función de diversas desigualdades" (Igareda y Cruells, 2014: 10).

En segundo lugar, debemos resaltar los compromisos del Estado español respecto a la garantía de los derechos de todas las personas (Sepúlveda, 2013) $y$, en particular, de las mujeres. En ese sentido, España, como Estado parte de la CEDAW, se ha comprometido a tomar "en todas las esferas, y en particular en las esferas política, social, económica y cultural, todas las medidas apropiadas, incluso de carácter legislativo, para asegurar el pleno desarrollo y adelanto de la mujer, con el objeto de garantizarle el ejercicio y el goce de los derechos humanos y las libertades fundamentales en igualdad de condiciones con el hombre" (artículo 3). Como se ha argumentado, la regulación de los trabajos de cuidados tiene un impacto de género y derechos que debe ser tenido en cuenta transversalmente a la hora de aprobar normas y políticas públicas.
Precisamente por ello, en tercer lugar, se hace referencia a un nuevo marco regulatorio que, bajo esas premisas, redistribuya la responsabilidad de cuidar, garantizando una sociedad más justa y equitativa, así como el bienestar y los derechos de todas las personas. Las propuestas concretas para articular un nuevo sistema de cuidados son tan variadas como las necesidades, problemas y desafíos a los que debe responder dicha regulación.

A modo de ejemplo cabe mencionar el reconocimiento de un derecho al cuidado, como una exigencia de Justicia social en el marco del Estado Social de Derecho (Igareda, 2012) o la adopción de normas específicas dirigidas a la redistribución de la responsabilidad de cuidar de una manera equitativa. Como apunta Frances Olsen (1990: 2):

Las normas antidiscriminatorias podrían requerir, por ejemplo, que el trabajo sea estructurado de manera tal que los trabajadores puedan dedicar períodos significativos de tiempo al cuidado de sus hijos sin perjudicar sus ingresos o carreras, o podría requerir la noción de valor comparable, es decir, que los trabajos -incluido el cuidado de los hijos- sean remunerados de acuerdo con la habilidad y responsabilidad que suponen.

En definitiva, como apunta Encarna Bodelón (2010a: 192), se trata de "hacer complejo el concepto de justicia e incluir la idea de cuidado", situando dicha noción histórica y socialmente y teniendo presente la diversidad de personas, necesidades y cuidados. 
BARTLETT, K. (1990): “Feminist legal methods”, Harvard Law Review, vol. 103, no4, págs. 829-888 [<http:// scholarship.law.duke.edu/cgi/viewcontent.cgi? article $=1119 \&$ context $=$ faculty_scholarship $\gg]$.

BELTRÁN, E.; y MAQUIEIRA, V. (eds.) (2005): Feminismos. Debates teóricos contemporáneos, Madrid, Alianza Editorial.

BENHABIB, S. (1992): "Una revisión del debate sobre las mujeres y la teoría moral”, Isegoría, no 6, págs. 37-63 [rhttp://isegoria.revistas.csic.es/index. php/isegoria/article/viewFile/323/324'].

BODELÓN, E. (2010a): “Derecho y justicia no androcéntricos", Quaderns de Psicologia, vol. 12, no 2, págs. 183-193 [‘http://www.raco. cat/index.php/QuadernsPsicologia/article/ viewFile/215016/285785>].

- (2010b): “Leyes de igualdad en Europa y transformaciones de la ciudadanía”, en HEIM, D.; y BODELÓN, E. (eds.), Género, Derecho e igualdad. Cambios en las estructuras jurídicas androcéntricas, vol. I, Barcelona, Grupo Antígona, págs. 9-27.

CARRASCO, C. (2006): “La paradoja del cuidado: necesario pero invisible", Revista de Economía Crítica, no 5, págs. 39-64 [<http://revistaeconomiacritica. org/sites/default/files/revistas/n5/2_ paradoja_del_cuidado.pdf $\rangle]$.

DE BEAUVOIR, S. (2002): El segundo sexo. Volumen I. Los hechos y los mitos, Madrid, Cátedra, serie Feminismos.

DELGADO, M.; ZAMORA, F.; y BARRIOS, L. (2006): “Déficit de fecundidad en España: factores demográficos que operan sobre una tasa muy inferior al nivel de reemplazo", Revista Española de Investigaciones Sociológicas, vol. 115, n- 6, págs. 197-222.
DÍAZ GARCíA, E. (1998): Curso de Filosofía del Derecho, Barcelona, Marcial Pons.

DÍAZ VELÁZQUEZ, E. (2010): “¿Políticas sociales de dependencia o de promoción de la autonomía?", Zerbitzuan, no 48, págs. 33-41 [<http://www.zerbitzuan.net/documentos/ zerbitzuan/Politicas\%20sociales\%20de\%20 dependencia.pdf $)$.

DURÁN, M. A. (2006): “Dependientes y cuidadores: el desafío de los próximos años", Revista del Ministerio de Trabajo e Inmigración, no 60, págs. 57-74 [<http://www.empleo.gob.es/es/ publica/pub_electronicas/destacadas/revista/ numeros/60/Esto4.pdf〉].

- (2001): “Con razón pero sin cuenta”, Cuenta y Razón, no 120 , págs. 1-8 [<http://digital.csic.es/ bitstream/10261/108799/1/Con\%2orazón,\%20 pero\%2osin\%2ocuenta-Revista\%2oCuenta\%20 y\%2oRazón.pdf〉].

ESPAÑA (2007): "Ley Orgánica 3/2007, de 22 de marzo, para la Igualdad Efectiva de Mujeres y Hombres", Boletín Oficial del Estado, ํㅜ 71, 23-3-07, págs. 12.611-12.645 [<https://www.boe.es/buscar/ doc.php?id=BOE-A-2007-6115'].

- (2006): “Ley 39/2006, de 14 de diciembre, de Promoción de la Autonomía Personal y Atención a las Personas en Situación de Dependencia", Boletín Oficial del Estado, n-299, 15-12-06, págs. 44.142-44.156 [rhttps://www.boe.es/boe/ dias/2006/12/15/pdfs/A44142-44156.pdf)].

- (1978): “Constitución Española”, Boletín Oficial del Estado, 29-12-1978, n- 311, págs. 29.31329.424 [ [hhttps://www.boe.es/buscar/act. php?id=BOE-A-1978-31229>].

ESPING-ANDERSEN, G. (coord.) (2013): El déficit de la natalidad en Europa. La singularidad del caso 
español, Barcelona, Obra Social La Caixa [rhttps://obrasocial.lacaixa.es/deployedfiles/ obrasocial/Estaticos/pdf/Estudios_sociales/ vol36_es.pdf〉].

FACIO, A. (1999): "Feminismo, género y patriarcado", FRIES, L.; y FACIO, A. (eds.), Género y Derecho, Santiago de Chile, LOM Ediciones; La Morada, págs. 21-60.

- (2004): “Metodología para el análisis de género de un proyecto de ley", Otras Miradas, vol. 4, no 1 , págs. 1-11 [<http://www.redalyc.org/ pdf/183/18340101.pdf>].

GILLIGAN, C. (2013): La ética del cuidado, Barcelona, Fundació Víctor Grifols i Lucas, serie Cuadernos de la Fundació Víctor Grifols i Lucas, $\mathrm{n}$ - 30 [<http://www.fundaciogrifols. org/documents/4662337/4689293/ cuaderno3o.pdf/91938ef9-d4do-4077-86001 cocd403c9ec)].

GUZMÁN, F.; MOSCOSO, M.; y TOBOSO, M. (2010): “Por qué la Ley de Dependencia no constituye un instrumento para la promoción de la autonomía personal”, Zerbitzuan, no 48, págs. 43-56 [khttp://www.zerbitzuan.net/documentos/ zerbitzuan/Ley\%2ode\%2odependencia\%20 no\%20constituye\%2oun\%2oinstrumento.pdf $>$ ].

HARTMANN, H. (1979): “Un matrimonio mal avenido: hacia una unión más progresiva entre marxismo y feminismo", Papers de la Fundació [Rafael Campalans], no 88 [/http://www.fcampalans. cat/archivos/papers/88.pdf)].

IGAREDA, N. (2012): "El derecho al cuidado en el Estado social de Derecho", Anuario de Filosofía del Derecho, nㅡㄹ 28, págs. 186-206.

IGAREDA, N.; y CRUELLS, M. (2014): “Críticas al derecho y el sujeto 'mujeres' y propuestas desde la jurisprudencia feminista", Cuadernos Electrónicos de Filosofía del Derecho, $\mathrm{n}-30$, págs. 1-16 [khttps://ojs.uv.es/index.php/CEFD/ article/viewFile/4107/4224'].

LAPORTA, E.; y DE LAS HERAS, S. (2014): "Una mirada desde el feminismo jurídico", Feminicidio.net [shttp:// www.feminicidio.net/articulo/una-miradadesde-el-feminismo-jur\% 3 CADdico〉].

MACKINNON, C. (1995): Hacia una teoría feminista del Estado, Madrid, Ediciones Cátedra, colección Feminismos.

MARTÍNEZ BUJÁN, R. (2011): “La reorganización de los cuidados familiares en un contexto de migración internacional", Cuadernos de Relaciones Laborales, vol. 29, $\mathrm{n}$ ำ1, págs. 93123 [<http://revistas.ucm.es/index.php/CRLA/ article/view/36187/35053'].

MARTÍNEZ VIRTO, L. (2010): “Gestión de los cuidados, desnacionalización y precariedad laboral: una perspectiva comparada", Alternativas, $\mathrm{n}^{-}$
17, págs. 221-235 [rhttp://rua.ua.es/dspace/ bitstream/10045/14305/1/Alternativas_17_12. pdf>].

MIER, I. et al. (2007): "Interpretando el cuidado. Por qué cuidan sólo las mujeres y qué podemos hacer para evitarlo", Zerbitzuan, no 42, págs. 29-38 [<http://www.zerbitzuan.net/documentos/ zerbitzuan/Interpretando\%2oel\%2ocuidado. pdf〉].

OFICINA DEL ALTO COMISIONADO DE NACIONES UNIDAS PARA LOS DERECHOS HUMANOS (2006): “El enfoque basado en los derechos humanos: definición y aspectos generales", en Preguntas frecuentes sobre el enfoque de derechos humanos en la cooperación para el desarrollo, Nueva York; Ginebra, Naciones Unidas [<http:// www.ohchr.org/Documents/Publications/ FAQsp.pdf〉].

OLSEN, F. (1990): “El sexo del Derecho”, en KAIRYS, D. (ed.), The Politics of Law, Nueva York, Panteón, págs. 452-467.

ONU (1979): Convención para la Eliminación de Todas las Formas de Discriminación contra la Mujer, Organización de las Naciones Unidas [<http:// www.ohchr.org/SP/Professionallnterest/ Pages/CEDAW.aspx>].

ONU (1994): Programa de Acción de la Conferencia Internacional sobre la Población y el Desarrollo, Organización de las Naciones Unidas [khttp:// www.unfpa.org/sites/default/files/pub-pdf/ icpd_spa.pdf>].

PATEMAN, C. (1996): "Críticas feministas a la dicotomía público/privado", en CASTELLS, C. (comp.): Perspectivas feministas en teoría política, Barcelona, Paidós, págs. 31-52.

PECES-BARBA, G. (1999): Curso de derechos fundamentales. Teoría general, Madrid, Universidad Carlos III de Madrid; Madrid, Boletín Oficial del Estado.

PÉREZ OROZCO, A. (2011): “Crisis multidimensional y sostenibilidad de la vida", Investigaciones Feministas, vol. 2, págs. 29-53 [shttp:// revistas.ucm.es/index.php/INFE/article/ view/38603/37328>].

PÉREZ OROZCO, A.; y LÓPEZ GIL, S. (2011): Desigualdades a flor de piel: cadenas globales de cuidados. Concreciones en el empleo de hogary articulaciones políticas, Santo Domingo, ONU Mujeres.

SEPÚLVEDA, M. (2013): Informe de la relatora especial sobre la extrema pobreza y los derechos humanos, Organización de las Naciones Unidas, A/68/293, 9 de agosto [khttp://ap.ohchr.org/ documents/dpage_s.aspx?s=41>].

TOBío, C. et al., (2010): El cuidado de las personas. Un reto para el siglo XXI, Barcelona, Fundació La Caixa. 